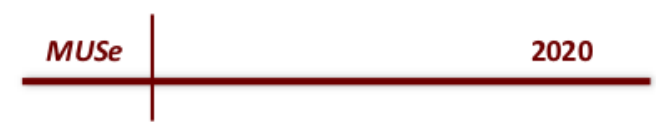

\title{
Lie Groups and Quantum Chromodynamics
}

\author{
Aaron C. H. Davey
}

\section{Introduction and History}

The purpose of this report is to obtain some insight into the subject of quantum field theory by way of a mathematical approach using group and Lie theory. Our goal is to bring together information from both mathematics and physics and unify it with a common simple language, which is not commonly done in most quantum physics publications.

We shall be looking into specific groups such as the special unitary group $S_{3}(\mathbb{C})$ and its Lie algebra $\mathfrak{s u}_{3}(\mathbb{C})$ using the Gell-Mann matrices, named after the physicist Murray Gell-Mann, as a representation. However, there is a section before this that is dedicated to reminding the reader on what exactly groups are and how they behave, and also to giving the definition and a summary of group representations.

An example of the lower dimensional case of $S U_{2}(\mathbb{C})$ is given with the Pauli matrices to give the reader an idea of what is to come and allows them to be in that mind frame once they jump to the more complex case later in the report. It is important to look at a simpler case with examples in order to understand anything at a higher level. The writer of this text has only a very basic understanding of classical and quantum physics, the latter by way of group theory and representations thanks to Peter Woit and his notes on the subject for an upcoming publication with Springer; this can be found in the reference section at the end of this report [14]. Woit's notes are very well

written and give a budding mathematician a workable avenue into quantum theory and quantum mechanics. Of course, it would at least if the mathematician has experience in abstract algebra with regard to subjects such as group, field and ring theory, as well as some experience with Lie groups and representation theory is likely very helpful.

Quantum field theory is a theory of the interactions between particles which represents the coming together of quantum mechanics and special relativity [10]. Quantum field theory has allowed physicists to tackle some of the biggest problems plaguing the subject during the twentieth century and allowed the formation of the Standard Model of particle physics, presumably the best chance of understanding everything in our universe. Major portions contributing to this are quantum electrodynamics (QED) and quantum chromodynamics (QCD), the latter being the subject of the last half of this report. QCD is the study of the strong interaction between the quarks of a nucleon and the gluons keeping the whole particle together.

Quantum chromodynamics is a non-abelian gauge theory with its symmetry group given as $S U_{3}(\mathbb{C})$; it is non-abelian because of the non-commutative property of our Lie group. A gauge theory is a certain type of field theory where the Lagrangian or Lagrangian density function is invariant under a continuous group, in our case the Lie group $S U_{3}(\mathbb{C})$, of local transformations [4].

Vol. 4(1) | DOI: https://doi.org/10.31542/muse.v4i1.881

MacEwan University Student eJournal | (C) 2020 under CC BY-NC | ISSN 2369-5617 


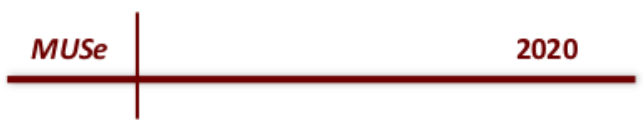

The Lagrangian density is a function of the fields in the system being explored; in the case of QCD, the Lagrangian is as follows below [6]:

$$
\mathcal{L}_{Q C D}=-\frac{1}{4} F_{\mu \nu}^{a} F^{a \mu \nu}-\sum_{f} \bar{q}_{f}\left[\gamma^{\mu} \frac{1}{i}\left(\partial_{\mu}-i g-i g \frac{\lambda_{a}}{2} A_{\mu}^{a}\right)+m_{f}\right] q_{f}
$$

where the index $a=1,2, \ldots, 8$ represents the eight colours of gluon fields, $\lambda_{a}$ represents the Gell-Mann matrices and the sum over $f$ is the summation over all different quark flavours [6].

In quantum field theory there are strict rules determining a viable framework. This includes a quantity being invariant under Lorentz transformations and the requirement of an invariant Lagrangian as mentioned previously. This Lorentz invariance is the other aspect of the theory of QCD and will not be discussed here; we will consider only the tools for the internal symmetries of the theory.

The Gell-Mann matrices that will be used in describing the mathematics behind our specific Lie groups are very important in physics. These matrices have connections to Murray Gell-Mann's proposed quark model and the strong force; more importantly they describe gluons, the particle responsible for the strong interaction between quarks and anti-quarks in a hadron [6]. It should

be noted that there are recommended prerequisites to obtain a good understanding of this report. These subjects include university-level courses in linear algebra, group theory and calculus. First and second year physics courses would be an asset to the latter half of the report on quantum chromodynamics; courses in quantum mechanics and particle physics would be beneficial but are in no way required.

\section{Background Information}

This research paper focuses on a few specific linear groups, or Lie groups, and their Lie algebras. We then use this Lie theory to look into a specific type of quantum field theory.

\subsection{Basic Group Theory and Representations}

First let us review the basic notions of a group. A group is a set $G$ with one operation, denoted as $*$ such that $G \times G \rightarrow G$; defined by $\left(g, g^{\prime}\right) \mapsto g * g^{\prime}$. All groups must adhere to the following axioms listed below:

- $g * h \in G$; for all $g, h \in G$; this is stated here for clarity but follows from the definition above.

- $(g * h) * k=g *(h * k)$; for all $g, h, k \in G$.

- There exists an element $e \in G$ such that $g * e=e * g=g$; for all $g \in G$.

- For each $g \in G$, there exists an element $g^{-1} \in G$ such that $g * g^{-1}=g^{-1} * g=e$.

Note also that the identity element and inverses are both unique. If $g, h \in G$ then $(g * h)^{-1}=$ $h^{-1} * g^{-1}$. 


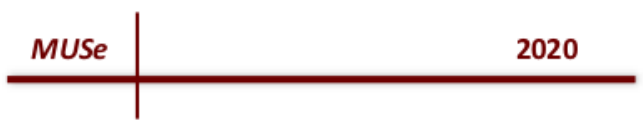

A representation $(\pi, V)$ of a group $G$ on a vector space $V$ is a homomorphism $\pi: G \rightarrow G L(V)$, defined by $g \mapsto \pi(g)$, where $G L(V)$ is the group of invertible linear maps from $V$ to $V$ [2]. We can rewrite this to suit the purposes of this paper by defining a representation $\left(\pi, V=\mathbb{C}^{n}\right)$ of the Lie group $S U_{n}(\mathbb{C})$ as a homomorphism $\pi: S U_{n}(\mathbb{C}) \rightarrow G L(n, V)$, defined by $M \mapsto \pi(M)$.

\subsection{Basic Lie Theory}

A Lie group is a smooth or differentiable manifold that obeys group properties [13]. It satisfies the condition that the group operations are differentiable [8]; in other words a Lie group is a smooth surface on which one can do calculus on the elements and can also have the group operations applied to them. A manifold is a topological space together with specific properties such as it locally resembles $\mathbb{R}^{n}[9]$. Knowing the precise definition of a manifold is not needed for us as we are working with specific Lie groups, but the interested reader may consult literature from sources such as MIT Open Courseware to learn about this. Examples of manifolds include the group $S U_{2}(\mathbb{C})$, which is isomorphic to a 3 -sphere and the group $U_{1}(\mathbb{C})$ creates a circle in the complex plane which is isomorphic to $\mathbb{R}^{2}$.

The specific Lie groups and Lie algebras we will be working with are outlined below where $\mathcal{M}_{n}(\mathbb{C})$ is the group of all $n \times n$ matrices, $U_{n}$ is the unitary group, $S U_{n}$ is the special unitary group and $\mathfrak{s u}_{\mathfrak{n}}$ is the Lie algebra of $S U_{n}$ [7]:

$$
\begin{gathered}
U_{n}(\mathbb{C})=\left\{M \in \mathcal{M}_{n}(\mathbb{C}) \mid M^{*} M=I_{n}\right\} \\
S U_{n}(\mathbb{C})=\left\{M \in U_{n}(\mathbb{C}) \mid \operatorname{det}(M)=1\right\} \\
\mathfrak{s u}_{\mathfrak{n}}(\mathbb{C})=\left\{A \in \mathcal{M}_{n}(\mathbb{C}) \mid A^{*}+A=0_{n}, \operatorname{tr}(A)=0\right\}
\end{gathered}
$$

Note that in the above groups $M^{*}=\bar{M}^{T}$, or in other words $M^{*}$ denotes the complex conjugate transpose of a matrix $M$. This is obtained by taking the complex conjugate of all entries and then transposing the matrix.

A Lie algebra $L$ is a vector space over a field $F$ (usually $F=\mathbb{C}$ ) that has a bracket operation called the Lie bracket and is denoted $[A, B]$; for all $A, B \in L$. The Lie algebra is closed under the Lie bracket which also obeys properties such as being anti-symmetric and bilinear [7]. Explicitly, anti-symmetry is defined as $[A, B]+[B, A]=0$. It also obeys the Jacobi identity which is expressed below:

$$
[A,[B, C]]+[[B,[C, A]]+[C,[A, B]]=0
$$

For a positive integer $n$, if we choose a subgroup of $G L_{n}(\mathbb{C})$, then the Lie algebra of this subgroup is a subspace of the vector space $\mathcal{M}_{n}(\mathbb{C})$ [7]. This comes with a matrix bracket of two matrices $A, B \in \mathcal{M}_{n}(\mathbb{C})$ called the commutator defined below:

$$
[A, B]=A B-B A
$$

As a side note, if the above relation was to equal zero for all elements of the Lie algebra, then the Lie algebra would be called abelian. 


\subsection{The Pauli Matrices}

The Pauli matrices are a common basis to use in the strange world of quantum mechanics, especially in regard to the Lie group $S U_{2}(\mathbb{C})$, since they are $2 \times 2$ matrices with complex entries [14]. They can easily be found by taking a generic element in $S_{2}$, which will be Hermitian and with a determinant equal to one, and expanding each entry into its general complex form, as shown below.

$$
M=\left[\begin{array}{cc}
w & z \\
-\bar{z} & \bar{w}
\end{array}\right]=\left[\begin{array}{cc}
a+i b & c+i d \\
-c+i d & a-i b
\end{array}\right]
$$

Now that a generic element is in this form, writing it in terms of the Pauli matrices is simple; however, the third matrix and constant are multiplied by the imaginary number $i$ for convenience in later calculations [14].

$$
M=a\left[\begin{array}{ll}
1 & 0 \\
0 & 1
\end{array}\right]+i b\left[\begin{array}{cc}
1 & 0 \\
0 & -1
\end{array}\right]+i c\left[\begin{array}{cc}
0 & i \\
-i & 0
\end{array}\right]+i d\left[\begin{array}{ll}
0 & 1 \\
1 & 0
\end{array}\right]
$$

We now have the three Pauli matrices and the $2 \times 2$ identity matrix, which in the order written above are $\sigma_{0}=I, \sigma_{3}, \sigma_{2}, \sigma_{1}$. These four matrices create a frequently used basis in which to express elements of $S U_{2}$ since the basis appears naturally and easily. The equation of an element $M \in S U_{2}(\mathbb{C})$ can be explicitly written out as a linear combination with the Pauli matrices and the identity matrix as a basis, as shown below:

$$
M=c_{0} I_{2}+c_{1} i \sigma_{1}+c_{2} i \sigma_{2}+c_{3} i \sigma_{3} ; c_{j} \in \mathbb{R}
$$

Next, we can use the Pauli matrices $\sigma_{j}$ where $j=1,2,3$, times $i$ to create a basis for the Lie algebra $\mathfrak{s u}_{2}(\mathbb{C})$ of $S U_{2}(\mathbb{C})$. A generic element $A \in \mathfrak{s u}_{2}$ can be written explicitly in terms of the Pauli matrices as shown below:

$$
\begin{gathered}
A=c_{1} i\left[\begin{array}{ll}
0 & 1 \\
1 & 0
\end{array}\right]+c_{2} i\left[\begin{array}{cc}
0 & i \\
-i & 0
\end{array}\right]+c_{3} i\left[\begin{array}{cc}
1 & 0 \\
0 & -1
\end{array}\right] \\
A=\left[\begin{array}{cc}
0 & i c_{1} \\
i c_{1} & 0
\end{array}\right]+\left[\begin{array}{cc}
0 & -c_{2} \\
c_{2} & 0
\end{array}\right]+\left[\begin{array}{cc}
i c_{3} & 0 \\
0 & -i c_{3}
\end{array}\right] \\
A=\left[\begin{array}{cc}
i c_{3} & -c_{2}+i c_{1} \\
c_{2}+i c_{1} & -i c_{3}
\end{array}\right]=\left[\begin{array}{cc}
i x & -\bar{z} \\
z & -i x
\end{array}\right] ; x \in \mathbb{R}, z \in \mathbb{C}
\end{gathered}
$$

Therefore, we can see that the trace of this element will be zero and that $\bar{A}^{T}+A=0$; these are the two properties needed for an element to be in $\mathfrak{s u}_{2}(\mathbb{C})$.

We can express the elements of the Lie algebra in a more general fashion using the Lie bracket commutation relation:

$$
\left[\sigma_{i}, \sigma_{j}\right]=\sigma_{i} \sigma_{j}-\sigma_{j} \sigma_{i}
$$

We can rewrite this bracket into the following form:

$$
\left[\sigma_{i}, \sigma_{j}\right]=i \sum_{k=1}^{3} \epsilon_{i j k} \sigma_{k}
$$




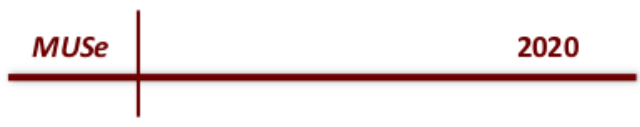

where $\epsilon_{i j k}$ denotes the structure constants, given below afterwards, and $\sigma_{j}$ denote the Pauli matrices; we assume they are the generators of $\mathfrak{s u}_{2}(\mathbb{C})$, but as we shall see later on with $\mathfrak{s u}_{3}$ it is not necessarily quite that simple.

The structure constants take on the values shown below:

$$
\epsilon_{i j k}= \begin{cases}-1, & \text { for }(i, j, k)=(1,3,2),(3,2,1),(2,1,3) \\ 0, & \text { for } i=j, i=k, j=k \\ +1, & \text { for }(i, j, k)=(1,2,3),(2,3,1),(3,1,2)\end{cases}
$$

We can now do something a little clever to obtain elements $M$ of $S U_{2}(\mathbb{C})$ if we have elements $A$ of $\mathfrak{s u}_{2}(\mathbb{C})$. Exponentiating a Lie algebra element will give us a Lie group element and therefore is a simple way of obtaining useful information [7].

The exponential of a matrix $X$ is given by the following formula:

$$
\exp (X)=I+X+\frac{X^{2}}{2 !}+\cdots+\frac{X^{n}}{n !}+\cdots
$$

An exponential map $\exp$ sends an element $A$ of the Lie algebra to an element $M$ of the Lie group written $\exp : \mathfrak{g} \rightarrow G$ or $\exp : \mathfrak{s u}_{2} \rightarrow S U_{2}$ [7].

$$
A=\left[\begin{array}{cc}
i c_{3} & -c_{2}+i c_{1} \\
c_{2}+i c_{1} & -i c_{3}
\end{array}\right] \mapsto M=\left[\begin{array}{cc}
e^{i c_{3}} & e^{-c_{2}+i c_{1}} \\
e^{c_{2}+i c_{1}} & e^{-i c_{3}}
\end{array}\right]
$$

We can see from above that $\operatorname{tr}(M)=e^{i c_{3}}+e^{-i c_{3}}$ and the determinant is equal to $\left|e^{i c_{3}}\right|^{2}+$ $\left|e^{c_{2}+i c_{1}}\right|^{2}=\left|e^{i x}\right|^{2}+\left|e^{z}\right|^{2}=1 ; x \in \mathbb{R}, z \in \mathbb{C}$.

Now we can move from working with the simpler case of the Pauli Matrices and $S U_{2}(\mathbb{C})$ to working with the more complicated case of the Gell-Mann matrices and $S U_{3}(\mathbb{C})$ in the next section.

\section{Gell-Mann Matrix Representation}

In this section, we introduce the Gell-Mann matrices and a few of their uses in physics. We will be working with $G L(n, V) \cong G L(n, \mathbb{C})$, where $G L(n, \mathbb{C})$ is the group of invertible $n \times n$ matrices with complex entries.

A representation $\pi$ of $S U_{3}(\mathbb{C})$ on $\mathbb{C}^{3}$ is a homomorphism $\pi: S U_{3}(\mathbb{C}) \rightarrow G L_{3}(\mathbb{C})$, defined by $M \mapsto \pi(M)$. For $\pi$ to be a group homomorphism we must be able to satisfy the property that for all $g, h \in G$ then $\pi(g h)=\pi(g) \pi(h)$. It is easy to see $\pi$ is indeed a homomorphism and that the group structure will be preserved since the matrices $\pi(M)$ for all $M \in S U_{3}$, will take values in $S U_{3} \subset G L_{3}$.

Each element in the group is assigned to an $n \times n$ matrix in such a way that these matrices behave in exactly the same way as the original group elements. Because the original group elements are also matrices, there are no "behavioural" changes, and in fact the matrix that is 
assigned to it is able to be expressed as a linear combination of the Gell-Mann matrix basis taking elements from $S U_{3}(\mathbb{C}) \subset G L_{3}(\mathbb{C})$.

It would also be correct to say that a group action on a vector space is called a representation of the group and in our case, an element $M \in S U_{3}$ acts on an element $x \in \mathbb{C}^{3}$ by $\pi(M)(x)=$ $M \cdot x=M x$; left matrix multiplication on a $3 \times 1$ vector with complex entries in $\mathbb{C}^{3}[2]$.

\subsection{Gell-Mann Matrices}

The Gell-Mann matrices are shown below [6]:

$$
\begin{gathered}
\lambda_{1}=\left[\begin{array}{lll}
0 & 1 & 0 \\
1 & 0 & 0 \\
0 & 0 & 0
\end{array}\right] \lambda_{2}=\left[\begin{array}{ccc}
0 & -i & 0 \\
i & 0 & 0 \\
0 & 0 & 0
\end{array}\right] \lambda_{3}=\left[\begin{array}{ccc}
1 & 0 & 0 \\
0 & -1 & 0 \\
0 & 0 & 0
\end{array}\right] \\
\lambda_{4}=\left[\begin{array}{lll}
0 & 0 & 1 \\
0 & 0 & 0 \\
1 & 0 & 0
\end{array}\right] \lambda_{5}=\left[\begin{array}{ccc}
0 & 0 & -i \\
0 & 0 & 0 \\
i & 0 & 0
\end{array}\right] \lambda_{6}=\left[\begin{array}{lll}
0 & 0 & 0 \\
0 & 0 & 1 \\
0 & 1 & 0
\end{array}\right] \\
\lambda_{7}=\left[\begin{array}{ccc}
0 & 0 & 0 \\
0 & 0 & -i \\
0 & i & 0
\end{array}\right] \lambda_{8}=\left[\begin{array}{ccc}
\frac{1}{\sqrt{3}} & 0 & 0 \\
0 & \frac{1}{\sqrt{3}} & 0 \\
0 & 0 & \frac{-2}{\sqrt{3}}
\end{array}\right]=\frac{1}{\sqrt{3}}\left[\begin{array}{ccc}
1 & 0 & 0 \\
0 & 1 & 0 \\
0 & 0 & -2
\end{array}\right]
\end{gathered}
$$

The Gell-Mann matrices are $3 \times 3$ Hermitian matrices with $\operatorname{tr}\left(\lambda_{j}\right)=0$ where $j=1,2, \ldots, 8$, and are outlined in detail in Section 4. Also, if any two Gell-Mann matrices are multiplied together, then their resulting product will have a trace equal to two times the Kronecker delta; in other words [12]:

$$
\operatorname{tr}\left(\lambda_{i} \lambda_{j}\right)=2 \delta_{i j} ; \text { where } \delta_{i j}= \begin{cases}0, & \text { if } i \neq j \\ 1, & \text { if } i=j\end{cases}
$$

The Kronecker delta becomes clear in an example shown below. We will be using Gell-Mann matrices $\lambda_{1}$ and $\lambda_{3}$ in the first part and $\lambda_{5}$ in the second part.

$$
\begin{aligned}
& {\left[\begin{array}{lll}
0 & 1 & 0 \\
1 & 0 & 0 \\
0 & 0 & 0
\end{array}\right]\left[\begin{array}{ccc}
1 & 0 & 0 \\
0 & -1 & 0 \\
0 & 0 & 0
\end{array}\right]=\left[\begin{array}{ccc}
0 & -1 & 0 \\
1 & 0 & 0 \\
0 & 0 & 0
\end{array}\right]} \\
& {\left[\begin{array}{ccc}
0 & 0 & -i \\
0 & 0 & 0 \\
i & 0 & 0
\end{array}\right]\left[\begin{array}{ccc}
0 & 0 & -i \\
0 & 0 & 0 \\
i & 0 & 0
\end{array}\right]=\left[\begin{array}{lll}
1 & 0 & 0 \\
0 & 0 & 0 \\
0 & 0 & 1
\end{array}\right]}
\end{aligned}
$$

The matrices used in the first example are both different and therefore $\delta_{13}=0$. Therefore, the trace of the product matrix must be zero which can be seen above. In the second example the matrices are both $\lambda_{5}$ and therefore $\delta_{55}=1$. Therefore, the trace of the product matrix must be two by definition. 


\subsection{Lie Groups and Algebras}

We can use the Gell-Mann matrices as a set of generators for the group $S U_{3}(\mathbb{C})$ which will be outlined in more detail as we go on. The Lie groups and Lie algebras that we will be focusing on include the special unitary group $S U_{3}(\mathbb{C})$ and its Lie algebra $\mathfrak{s u}_{3}(\mathbb{C})$, as well as the unitary group $U_{3}(\mathbb{C})$ and the group of all $3 \times 3$ matrices $\mathcal{M}_{3}(\mathbb{C})$, shown below [7]:

$$
\begin{gathered}
U_{3}(\mathbb{C})=\left\{M \in \mathcal{M}_{3}(\mathbb{C}) \mid M^{*} M=I_{3}\right\} \\
S U_{3}(\mathbb{C})=\left\{M \in U_{3}(\mathbb{C}) \mid \operatorname{det}(M)=1\right\} \\
\mathfrak{s u}_{3}(\mathbb{C})=\left\{A \in \mathcal{M}_{3}(\mathbb{C}) \mid A^{*}+A=0_{3}, \operatorname{tr}(A)=0\right\}
\end{gathered}
$$

\subsubsection{Lie Algebra and the Lie Bracket}

As we shall see shortly, we can use the Gell-Mann matrices as a basis for $\mathfrak{s u}_{3}(\mathbb{C})$ which means each element of the Lie algebra can be written as a linear combination of the Gell-Mann matrices. Furthermore, these matrices must obey the commutation relations of the Lie bracket.

$$
\left[\lambda_{i}, \lambda_{j}\right]=\lambda_{i} \lambda_{j}-\lambda_{j} \lambda_{i} ; \forall i, j \in\{1,2, \ldots, 8\}
$$

Let us consider an example by picking two Gell-Mann matrices, say $\lambda_{2}$ and $\lambda_{4}$. Then we have the following below:

$$
\begin{gathered}
{\left[\lambda_{2}, \lambda_{4}\right]=\lambda_{2} \lambda_{4}-\lambda_{4} \lambda_{2}} \\
=\left[\begin{array}{ccc}
0 & -i & 0 \\
i & 0 & 0 \\
0 & 0 & 0
\end{array}\right]\left[\begin{array}{lll}
0 & 0 & 1 \\
0 & 0 & 0 \\
1 & 0 & 0
\end{array}\right]-\left[\begin{array}{lll}
0 & 0 & 1 \\
0 & 0 & 0 \\
1 & 0 & 0
\end{array}\right]\left[\begin{array}{ccc}
0 & -i & 0 \\
i & 0 & 0 \\
0 & 0 & 0
\end{array}\right] \\
=\left[\begin{array}{lll}
0 & 0 & 0 \\
0 & 0 & i \\
0 & 0 & 0
\end{array}\right]-\left[\begin{array}{ccc}
0 & 0 & 0 \\
0 & 0 & 0 \\
0 & -i & 0
\end{array}\right]=\left[\begin{array}{lll}
0 & 0 & 0 \\
0 & 0 & i \\
0 & i & 0
\end{array}\right]=i\left[\begin{array}{lll}
0 & 0 & 0 \\
0 & 0 & 1 \\
0 & 1 & 0
\end{array}\right]=i \lambda_{6}
\end{gathered}
$$

It is easy to see that this matrix is also an element of $\mathfrak{s u}_{3}$, therefore, at least for this case, the commutation relation works out. It will be left as an exercise to the reader to check the commutator bracket for different combinations of the Gell-Mann matrices.

We can now look deeper into the fact that every element of $\mathfrak{s u}_{3}(\mathbb{C})$ can be written as a linear combination of the Gell-Mann matrices. The Lie bracket above generates another element of the Lie algebra; this is clear since the Lie bracket is closed in any Lie algebra.

We may use this fact to equate the Lie bracket with a linear combination of the Gell-Mann matrices. These are multiplied further by the structure constants, denoted $f^{i j k}$ and by the imaginary number $i$.

Therefore, we have the following equation for the Lie algebra $\mathfrak{s u}_{3}(\mathbb{C})$ : 


$$
\left[\lambda_{i}, \lambda_{j}\right]=i \sum_{k=1}^{8} f^{i j k} \lambda_{k}
$$

The structure constants have values shown below; all others not related to these by permutation of the indices are equal to zero:

$$
\begin{gathered}
f^{123}=1 \\
f^{147}=-f^{156}=f^{246}=f^{257}=f^{345}=-f^{367}=\frac{1}{2} \\
f^{458}=f^{678}=\frac{\sqrt{3}}{2}
\end{gathered}
$$

Let us now continue with our example from above. We know that our bracket calculation gives the following expression:

$$
\left[\lambda_{2}, \lambda_{4}\right]=\lambda_{2} \lambda_{4}-\lambda_{4} \lambda_{2}=i \lambda_{6}
$$

Now, let us use the other (possibly equivalent) relation and discover if we obtain the same answer.

$$
\begin{gathered}
i \sum_{k=1}^{8} f^{i j k} \lambda_{k}=i \sum_{k=1}^{8} f^{24 k} \lambda_{k} \\
=i f^{241} \lambda_{1}+i f^{242} \lambda_{2}+i f^{243} \lambda_{3}+i f^{244} \lambda_{4}+i f^{245} \lambda_{5}+i f^{246} \lambda_{6}+i f^{247} \lambda_{7}+i f^{248} \lambda_{8} \\
=i(0) \lambda_{1}+i(0) \lambda_{2}+i(0) \lambda_{3}+i(0) \lambda_{4}+i(0) \lambda_{5}+i\left(\frac{1}{2}\right) \lambda_{6}+i(0) \lambda_{7}+i(0) \lambda_{8} \\
i \sum_{k=1}^{8} f^{24 k} \lambda_{k}=i\left(\frac{1}{2}\right) \lambda_{6}
\end{gathered}
$$

Finally, we can put the two results together below and see that the two results differ only by $\frac{1}{2}$ :

$$
\begin{gathered}
{\left[\lambda_{2}, \lambda_{4}\right]=\lambda_{2} \lambda_{4}-\lambda_{4} \lambda_{2}=i \lambda_{6}} \\
i \sum_{k=1}^{8} f^{24 k} \lambda_{k}=\frac{i}{2} \lambda_{6}
\end{gathered}
$$

To correct this, instead of simply using the Gell-Mann matrices as generators for $\mathfrak{s u}_{3}$, we should be using a generator denoted $g_{i}=\frac{\lambda_{i}}{2}$. If we use this new generator and do the above calculations again, we find that they produce precisely the same element:

$$
\left[g_{i}, g_{j}\right]=\left[\frac{\lambda_{2}}{2}, \frac{\lambda_{4}}{2}\right]=\frac{\lambda_{2}}{2} \frac{\lambda_{4}}{2}-\frac{\lambda_{4}}{2} \frac{\lambda_{2}}{2}=\frac{i}{4} \lambda_{6}
$$




$$
\begin{array}{r|r}
\text { Muse } & 2020 \\
\hline & \\
i \sum_{k=1}^{8} f^{24 k} g_{k}=\frac{i}{2} \sum_{k=1}^{8} f^{24 k} \lambda_{k}=\frac{i}{4} \lambda_{6}
\end{array}
$$

The reader, as an exercise, may check that this new generator will also hold for any combination of Gell-Mann matrices, in both cases producing the same element of the Lie algebra. However to keep things simple, the rest of the paper will assume the generators to be $\lambda_{i}$ and not $g_{i}=\frac{\lambda_{i}}{2}$ since it is sufficient for our purposes outside these commutation relations.

\subsubsection{Elements of $S U_{3}(\mathbb{C})$ and $\mathfrak{s u}_{3}(\mathbb{C})$}

Since the Gell-Mann matrices can be used to generate elements of the Lie group $S_{3}$ analogous to the Pauli matrices and $S U_{2}$, then we should be able to use the Gell-Mann matrices as a basis for its Lie algebra $\mathfrak{s u}_{3}$ and exponentiate from there. Let us first start with a generic element $A$ of $\mathfrak{s u}_{3}(\mathbb{C})$ :

$$
A=\left[\begin{array}{ccc}
i v & x & y \\
-\bar{x} & i v & z \\
-\bar{y} & -\bar{z} & i(-2 v)
\end{array}\right] ; v \in \mathbb{R} ; x, y, z \in \mathbb{C}
$$

It can be easily checked that the trace of $A$ is zero and that $A^{*}+A=0$, especially when each element is expanded into its complex form, as shown below:

$$
\begin{aligned}
A & =\left[\begin{array}{ccc}
i c_{8}+i c_{3} & c_{2}+i c_{1} & c_{5}+i c_{4} \\
-c_{2}+i c_{1} & i c_{8}-i c_{3} & c_{7}+i c_{6} \\
-c_{5}+i c_{4} & -c_{7}+i c_{6} & -2 i c_{8}
\end{array}\right] ; c_{1}, c_{2}, \ldots, c_{8} \in \mathbb{R} \\
A^{*} & =\left[\begin{array}{ccc}
-i c_{8}-i c_{3} & -c_{2}-i c_{1} & -c_{5}-i c_{4} \\
c_{2}-i c_{1} & -i c_{8}+i c_{3} & -c_{7}-i c_{6} \\
c_{5}-i c_{4} & c_{7}-i c_{6} & 2 i c_{8}
\end{array}\right] ; c_{1}, c_{2}, \ldots, c_{8} \in \mathbb{R}
\end{aligned}
$$

It should be noted that the above matrix $A^{*}$ is equal to $-A$ and this is a special property: the matrix $A$ is called skew-Hermitian. Therefore, the Lie algebra $\mathfrak{s u}_{3}$ of $S U_{3}$ is equal to the space of skew-Hermitian matrices. The next step is to express this element in terms of the Gell-Mann matrices, and it can be written out explicitly by:

$$
\begin{aligned}
& A=i c_{1}\left[\begin{array}{lll}
0 & 1 & 0 \\
1 & 0 & 0 \\
0 & 0 & 0
\end{array}\right]+i c_{2}\left[\begin{array}{ccc}
0 & -i & 0 \\
i & 0 & 0 \\
0 & 0 & 0
\end{array}\right]+i c_{3}\left[\begin{array}{ccc}
1 & 0 & 0 \\
0 & -1 & 0 \\
0 & 0 & 0
\end{array}\right]+i c_{4}\left[\begin{array}{ccc}
0 & 0 & 1 \\
0 & 0 & 0 \\
1 & 0 & 0
\end{array}\right] \\
& +i c_{5}\left[\begin{array}{ccc}
0 & 0 & -i \\
0 & 0 & 0 \\
i & 0 & 0
\end{array}\right]+i c_{6}\left[\begin{array}{ccc}
0 & 0 & 0 \\
0 & 0 & 1 \\
0 & 1 & 0
\end{array}\right]+i c_{7}\left[\begin{array}{ccc}
0 & 0 & 0 \\
0 & 0 & -i \\
0 & i & 0
\end{array}\right]+i \frac{c_{8}}{\sqrt{3}}\left[\begin{array}{ccc}
1 & 0 & 0 \\
0 & 1 & 0 \\
0 & 0 & -2
\end{array}\right] \\
& A=i c_{1} \lambda_{1}+i c_{2} \lambda_{2}+i c_{3} \lambda_{3}+i c_{4} \lambda_{4}+i c_{5} \lambda_{5}+i c_{6} \lambda_{6}+i c_{7} \lambda_{7}+i c_{8} \lambda_{8}
\end{aligned}
$$

We can see each matrix $\lambda_{i}$ is linearly independent from another matrix $\lambda_{j}$. Therefore, no one matrix can be written as a linear combination of the others. Also, multiplying each $\lambda_{i}$ by the 


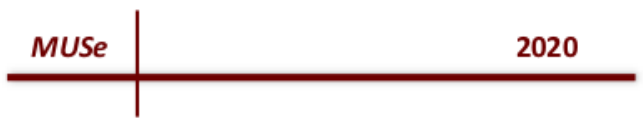

imaginary number $i$ is necessary for the matrices to be generators of the elements of $\mathfrak{s u}_{3}(\mathbb{C})$ just as it was to multiply each Pauli matrix $\sigma_{j}$ by $i$ for $\mathfrak{s u}_{2}(\mathbb{C})$.

Recall the definition of a matrix exponential found in equation 2.15 , shown below:

$$
\exp (X)=\sum_{n=0}^{\infty} \frac{X^{n}}{n !}=I+X+\frac{X^{2}}{2 !}+\frac{X^{3}}{3 !}+\cdots
$$

which we can also equate to a Taylor series expansion; in this case for the exponential function of a matrix [7]. Next, let us create an exponential map exp $: \mathfrak{s u}_{3}(\mathbb{C}) \rightarrow S U_{3}(\mathbb{C})$ that is defined by $A \mapsto \exp (A)=M$. Therefore, we can find elements in $S U_{3}$ by exponentiating elements of $\mathfrak{s u}_{3}$. Let us express an element $A \in \mathfrak{s u}_{3}$ by:

$$
A=\sum_{n=1}^{8} i c_{i} \lambda_{i}
$$

which can be expanded in the same way as in equation 3.23 :

$$
A=i c_{1} \lambda_{1}+i c_{2} \lambda_{2}+i c_{3} \lambda_{3}+i c_{4} \lambda_{4}+i c_{5} \lambda_{5}+i c_{6} \lambda_{6}+i c_{7} \lambda_{7}+i c_{8} \lambda_{8}
$$

Then, we can take our exponential function of the element $A$, defined by:

$$
\exp (A)=\exp \left(\sum_{n=1}^{8} i c_{i} \lambda_{i}\right)
$$

which takes $e$ and raises it to the power of the entire linear combination giving our new element $M \in S U_{3}$ where the Gell-Mann matrices are the generators:

$$
\begin{aligned}
M & =e^{i c_{1} \lambda_{1}+i c_{2} \lambda_{2}+i c_{3} \lambda_{3}+i c_{4} \lambda_{4}+i c_{5} \lambda_{5}+i c_{6} \lambda_{6}+i c_{7} \lambda_{7}+i c_{8} \lambda_{8}} \\
& =e^{i c_{1} \lambda_{1}} e^{i c_{2} \lambda_{2}} e^{i c_{3} \lambda_{3}} e^{i c_{4} \lambda_{4}} e^{i c_{5} \lambda_{5}} e^{i c_{6} \lambda_{6}} e^{i c_{7} \lambda_{7}} e^{i c_{8} \lambda_{8}}
\end{aligned}
$$

Next, for reasons concerning aesthetics and convention, let each $c_{i}=\theta_{i}$; for all $i \in\{1,2, \ldots, 8\}$. We can write an element $M \in S U_{3}(\mathbb{C})$ as a linear combination of matrices with entries that are possibly complex and in terms of sine and cosine. Therefore, an element $M \in S U_{3}$ can be written as follows below:

$$
\begin{aligned}
& M=\left[\begin{array}{ccc}
\cos \left(\theta_{1}\right) & i \sin \left(\theta_{1}\right) & 0 \\
i \sin \left(\theta_{1}\right) & \cos \left(\theta_{1}\right) & 0 \\
0 & 0 & 1
\end{array}\right]\left[\begin{array}{ccc}
\cos \left(\theta_{2}\right) & \sin \left(\theta_{2}\right) & 0 \\
-\sin \left(\theta_{2}\right) & \cos \left(\theta_{2}\right) & 0 \\
0 & 0 & 1
\end{array}\right]\left[\begin{array}{ccc}
e^{i \theta_{3}} & 0 & 0 \\
0 & e^{-i \theta_{3}} & 0 \\
0 & 0 & 1
\end{array}\right]\left[\begin{array}{ccc}
\cos \left(\theta_{4}\right) & 0 & i \sin \left(\theta_{4}\right) \\
0 & 1 & 0 \\
i \sin \left(\theta_{4}\right) & 0 & \cos \left(\theta_{4}\right)
\end{array}\right] * \\
& *\left[\begin{array}{ccc}
\cos \left(\theta_{5}\right) & 0 & \sin \left(\theta_{5}\right) \\
0 & 1 & 0 \\
-\sin \left(\theta_{5}\right) & 0 & \cos \left(\theta_{5}\right)
\end{array}\right]\left[\begin{array}{ccc}
1 & 0 & 0 \\
0 & \cos \left(\theta_{6}\right) & i \sin \left(\theta_{6}\right) \\
0 & i \sin \left(\theta_{6}\right) & \cos \left(\theta_{6}\right)
\end{array}\right]\left[\begin{array}{ccc}
1 & 0 & 0 \\
0 & \cos \left(\theta_{7}\right) & \sin \left(\theta_{7}\right) \\
0 & -\sin \left(\theta_{7}\right) & \cos \left(\theta_{7}\right)
\end{array}\right]\left[\begin{array}{ccc}
e^{\frac{i \theta_{8}}{\sqrt{3}}} & 0 & 0 \\
0 & e^{\frac{i \theta_{8}}{\sqrt{3}}} & 0 \\
0 & 0 & e^{\frac{-2 i \theta_{8}}{\sqrt{3}}}
\end{array}\right]
\end{aligned}
$$

It can be shown that each matrix component making up the element $M$ has determinant equal to one. By linear algebraic properties of determinants, if $A$ and $B$ are square matrices of the same 
size, then we have that $\operatorname{det}(A) \cdot \operatorname{det}(B)=\operatorname{det}(A B)$. Therefore, since all matrix components are $3 \times 3$ square matrices being multiplied together, then we have that $\operatorname{det}(M)=1^{8}=1$.

Let us digress for a moment and consider another Lie group, $\mathrm{SO}_{3}(\mathbb{R})$ : the special orthogonal group of $3 \times 3$ matrices with real entries:

$$
S O_{3}(\mathbb{R})=\left\{M \in G L_{3}(\mathbb{R}) \mid M^{T} M=I_{3}, \operatorname{det}(M)=1\right\}
$$

This group looks very similar to $S U_{3}$, other than its elements existing in $G L_{3}(\mathbb{R})$ and the lack of complex numbers. However, if we were to take all matrix elements $M \in S U_{3}$ that contained only real entries with determinant equal to one, we would get exactly $\mathrm{SO}_{3}$. Therefore, we can say that $S_{3}(\mathbb{R}) \subset S U_{3}(\mathbb{C})$ and we can further extend this to say that $S O_{n} \subset S U_{n}$.

A generic element $M \in S U_{3}(\mathbb{C})$ in this form can be obtained from simple matrix multiplication and will be left to the reader as an exercise. The reader can also take (as an exercise) any combination of components from our equation below and take the rest to have constants $\theta_{i}$ equal to zero and verify if the resulting matrix is still an element of $S_{3}$.

$$
M=e^{i \theta_{1} \lambda_{1}} e^{i \theta_{2} \lambda_{2}} e^{i \theta_{3} \lambda_{3}} e^{i \theta_{4} \lambda_{4}} e^{i \theta_{5} \lambda_{5}} e^{i \theta_{6} \lambda_{6}} e^{i \theta_{7} \lambda_{7}} e^{i \theta_{8} \lambda_{8}}
$$

Let us perform the following matrix multiplication to illustrate how one may find some element of $\mathrm{SU}_{3}$ :

$$
\begin{aligned}
N & =\left[\begin{array}{ccc}
\cos \left(\theta_{1}\right) & i \sin \left(\theta_{1}\right) & 0 \\
i \sin \left(\theta_{1}\right) & \cos \left(\theta_{1}\right) & 0 \\
0 & 0 & 1
\end{array}\right]\left[\begin{array}{ccc}
\cos \left(\theta_{2}\right) & \sin \left(\theta_{2}\right) & 0 \\
-\sin \left(\theta_{2}\right) & \cos \left(\theta_{2}\right) & 0 \\
0 & 0 & 1
\end{array}\right] \\
& =\left[\begin{array}{ccc}
\cos \left(\theta_{1}\right) \cos \left(\theta_{2}\right)-i \sin \left(\theta_{1}\right) \sin \left(\theta_{2}\right) & \cos \left(\theta_{1}\right) \sin \left(\theta_{2}\right)+i \sin \left(\theta_{1}\right) \cos \left(\theta_{2}\right) & 0 \\
-\left(\cos \left(\theta_{1}\right) \sin \left(\theta_{2}\right)-i \sin \left(\theta_{1}\right) \cos \left(\theta_{2}\right)\right) & \cos \left(\theta_{1}\right) \cos \left(\theta_{2}\right)+i \sin \left(\theta_{1}\right) \sin \left(\theta_{2}\right) & 0 \\
0 & 0 & 1
\end{array}\right]
\end{aligned}
$$

The matrix $N$ above is obtained from the general matrix $M$ by letting our constants $c_{i}=0$, for all $i \in\{3,4, \ldots, 8\}$ in Equation 3.28. From the previously mentioned determinant property, this matrix has determinant equal to one, for all $\theta_{1}, \theta_{2}$ from $0 \rightarrow 2 \pi$. Therefore, we can pick two angles to illustrate, for example $\theta_{1}=\frac{\pi}{4}$ and $\theta_{2}=\frac{\pi}{3}$, which gives us the matrix:

$$
N=\left[\begin{array}{ccc}
\left(\frac{1}{\sqrt{2}}\right)\left(\frac{1}{2}\right)-i\left(\frac{1}{\sqrt{2}}\right)\left(\frac{\sqrt{3}}{2}\right) & \left.\left(\frac{1}{\sqrt{2}}\right)\left(\frac{\sqrt{3}}{2}\right)+i\left(\frac{1}{\sqrt{2}}\right)\left(\frac{1}{2}\right)\right) & 0 \\
-\left(\left(\frac{1}{\sqrt{2}}\right)\left(\frac{\sqrt{3}}{2}\right)-i\left(\frac{1}{\sqrt{2}}\right)\left(\frac{1}{2}\right)\right) & \left(\frac{1}{\sqrt{2}}\right)\left(\frac{1}{2}\right)+i\left(\frac{1}{\sqrt{2}}\right)\left(\frac{\sqrt{3}}{2}\right) & 0 \\
0 & 0 & 1
\end{array}\right]=\left[\begin{array}{ccc}
\frac{1}{2 \sqrt{2}}-i \frac{\sqrt{3}}{2 \sqrt{2}} & \frac{\sqrt{3}}{2 \sqrt{2}}+i \frac{1}{2 \sqrt{2}} & 0 \\
-\left(\frac{\sqrt{3}}{2 \sqrt{2}}-i \frac{1}{2 \sqrt{2}}\right) & \frac{1}{2 \sqrt{2}}+i \frac{\sqrt{3}}{2 \sqrt{2}} & 0 \\
0 & 0 & 1
\end{array}\right]
$$

We can express the above matrix in such a way to achieve a sense of clarity and to make an important observation that will be discussed below.

$$
N=\left[\begin{array}{ccc}
a-i b & b+i a & 0 \\
-(b-i a) & a+i b & 0 \\
0 & 0 & 1
\end{array}\right]=\left[\begin{array}{ccc}
u & v & 0 \\
-\bar{v} & \bar{u} & 0 \\
0 & 0 & 1
\end{array}\right] ; u, v \in \mathbb{C}
$$




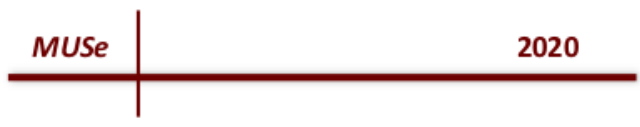

When taking the determinant of this matrix we obtain a $2 \times 2$ matrix with the exact form of an element of $S U_{2}(\mathbb{C})$ in Equation 3.33 below:

$$
\begin{gathered}
\operatorname{det}(N)=0\left|\begin{array}{cc}
v & 0 \\
\bar{u} & 0
\end{array}\right|-0\left|\begin{array}{cc}
u & 0 \\
-\bar{v} & 0
\end{array}\right|+1\left|\begin{array}{cc}
u & v \\
-\bar{v} & \bar{u}
\end{array}\right| \\
\operatorname{det}(N)=\left|\begin{array}{cc}
u & v \\
-\bar{v} & \bar{u}
\end{array}\right|
\end{gathered}
$$

Therefore, $\operatorname{det}(N)=|u|^{2}+|v|^{2}=\left(a^{2}+b^{2}\right)+\left(a^{2}+b^{2}\right)=1$; where $a=\frac{1}{2 \sqrt{2}}, b=\frac{\sqrt{3}}{2 \sqrt{2}}$. It would not be unreasonable to assume that any combination of matrices taken within our general element $M$ to construct some other matrix, in such a way as we did above, will be readily reduced to a similar form shown in Equation 3.31.

\section{Gell-Mann Matrix Properties}

This section includes information that has been and will be used in the various sections, as well as some added results that are interesting in their own right and could be used as a reference for related studies in linear algebra and Lie theory. In particular, several properties of the Gell-Mann matrices are presented, which in current literature tend to be under emphasized in the view of this author.

\section{Gell-Mann Matrix $\lambda_{1}$}

$$
\lambda_{1}=\left[\begin{array}{lll}
0 & 1 & 0 \\
1 & 0 & 0 \\
0 & 0 & 0
\end{array}\right]
$$

The first Gell-Mann matrix is a $3 \times 3$ square matrix. It is symmetric due to $\lambda_{1}=\left(\lambda_{1}\right)^{T}$ and it is Hermitian due to $\lambda_{1}=\left(\lambda_{1}\right)^{*}$. The determinant of $\lambda_{1}$ is equal to zero since it contains a row comprised of all zeros and the trace of $\lambda_{1}$ is also equal to zero.

Each of the three column vectors comprising $\lambda_{1}$ are $\overrightarrow{v_{1}}=(0,1,0), \overrightarrow{v_{2}}=(1,0,0)$ and $\overrightarrow{v_{3}}=$ $(0,0,0)$. These do not span $\mathbb{R}^{3}$ and since we have that $\overrightarrow{v_{3}}=\overrightarrow{0}$, these vectors are linearly dependent. The dimension of the column space and row space of $\lambda_{1}$ are both equal to the number of leading entries in the matrix which is 2 ; therefore the rank of $\lambda_{1}$ is also 2 .

To compute the eigenvalues we have the following:

$$
\begin{aligned}
\operatorname{det}\left(\varphi I-\lambda_{1}\right) & =\varphi\left|\begin{array}{ll}
\varphi & 0 \\
0 & \varphi
\end{array}\right|-(-1)\left|\begin{array}{cc}
-1 & 0 \\
0 & \varphi
\end{array}\right|+0\left|\begin{array}{cc}
-1 & \varphi \\
0 & 0
\end{array}\right| \\
& =\varphi\left(\varphi^{2}\right)+(-\varphi)+0 \\
& =\varphi^{3}-\varphi \\
& =\varphi\left(\varphi^{2}-1\right) \\
& =0
\end{aligned}
$$




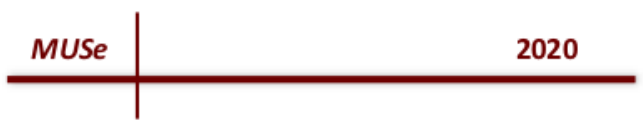

Therefore, the roots or eigenvalues of this equation are $\varphi=-1,0,1$.

Finally, we say that $\vec{x} \in \mathbb{R}^{3}$, where $\vec{x} \neq \overrightarrow{0}$, is an eigenvector of $\lambda_{1}$ if it satisfies the equation $\lambda_{1} \vec{x}=\varphi \vec{x}$. We may set this equation up in the form of matrices as follows:

$$
\left[\begin{array}{lll}
0 & 1 & 0 \\
1 & 0 & 0 \\
0 & 0 & 0
\end{array}\right]\left[\begin{array}{l}
x_{1} \\
x_{2} \\
x_{3}
\end{array}\right]=\varphi\left[\begin{array}{l}
x_{1} \\
x_{2} \\
x_{3}
\end{array}\right]
$$

Therefore, the solutions or eigenvectors satisfying this relation for $\varphi=-1,0,1$, respectively, are as follows:

$$
\overrightarrow{x_{1}}=\left[\begin{array}{c}
-1 \\
1 \\
0
\end{array}\right], \overrightarrow{x_{2}}=\left[\begin{array}{l}
0 \\
0 \\
1
\end{array}\right], \overrightarrow{x_{3}}=\left[\begin{array}{l}
1 \\
1 \\
0
\end{array}\right]
$$

\section{Gell-Mann Matrix $\lambda_{2}$}

The second Gell-Mann matrix is a $3 \times 3$ square matrix that is antisymmetric due to $\lambda_{2}=-\left(\lambda_{2}\right)^{T}$ and it is Hermitian due to $\lambda_{2}=\left(\lambda_{2}\right)^{*}$.

$$
\lambda_{2}=\left[\begin{array}{ccc}
0 & -i & 0 \\
i & 0 & 0 \\
0 & 0 & 0
\end{array}\right]
$$

The determinant of $\lambda_{2}$ is equal to zero since it contains a row comprised of all zeros and the trace of $\lambda_{2}$ is also equal to zero.

Each of the three column vectors comprising $\lambda_{2}$ are $\overrightarrow{v_{1}}=(0, i, 0), \overrightarrow{v_{2}}=(-i, 0,0)$ and $\overrightarrow{v_{3}}=$ $(0,0,0)$. These do not span $\mathbb{R}^{3}$ and since we have that $\overrightarrow{v_{3}}=\overrightarrow{0}$, these vectors are linearly dependent. The dimension of the column space and row space of $\lambda_{2}$ are both equal to the number of leading entries in the matrix which is 2 ; therefore the rank of $\lambda_{2}$ is also 2 .

To compute the eigenvalues we have the following:

$$
\begin{aligned}
\operatorname{det}\left(\varphi I-\lambda_{2}\right) & =\varphi\left|\begin{array}{ll}
\varphi & 0 \\
0 & \varphi
\end{array}\right|-i\left|\begin{array}{cc}
-i & 0 \\
0 & \varphi
\end{array}\right|+0\left|\begin{array}{cc}
-i & \varphi \\
0 & 0
\end{array}\right| \\
& =\varphi\left(\varphi^{2}\right)+i(i \varphi)+0 \\
& =\varphi^{3}-\varphi \\
& =\varphi\left(\varphi^{2}-1\right) \\
& =0
\end{aligned}
$$

Therefore, the roots or eigenvalues of this equation are $\varphi=-1,0,1$.

Finally, we say that $\vec{x} \in \mathbb{R}^{3}$, where $\vec{x} \neq \overrightarrow{0}$, is an eigenvector of $\lambda_{2}$ if it satisfies the equation $\lambda_{2} \vec{x}=\varphi \vec{x}$. We may set this equation up in the form of matrices as follows: 


$$
\left[\begin{array}{ccc}
0 & -i & 0 \\
i & 0 & 0 \\
0 & 0 & 0
\end{array}\right]\left[\begin{array}{l}
x_{1} \\
x_{2} \\
x_{3}
\end{array}\right]=\varphi\left[\begin{array}{l}
x_{1} \\
x_{2} \\
x_{3}
\end{array}\right]
$$

Therefore, the solutions or eigenvectors satisfying this relation for $\varphi=-1,0,1$, respectively, are as follows:

$$
\overrightarrow{x_{1}}=\left[\begin{array}{l}
i \\
1 \\
0
\end{array}\right], \overrightarrow{x_{2}}=\left[\begin{array}{l}
0 \\
0 \\
1
\end{array}\right], \overrightarrow{x_{3}}=\left[\begin{array}{c}
-i \\
1 \\
0
\end{array}\right]
$$

\section{Gell-Mann Matrix $\lambda_{3}$}

The third Gell-Mann matrix is a $3 \times 3$ square matrix that is diagonal due to every entry being zero other than on the main diagonal and it is Hermitian due to $\lambda_{3}=\left(\lambda_{3}\right)^{*}$.

$$
\lambda_{3}=\left[\begin{array}{ccc}
1 & 0 & 0 \\
0 & -1 & 0 \\
0 & 0 & 0
\end{array}\right]
$$

The determinant of $\lambda_{3}$ is equal to zero since it contains a row comprised of all zeros and the trace of $\lambda_{3}$ is also equal to zero.

Each of the three column vectors comprising $\lambda_{3}$ are $\overrightarrow{v_{1}}=(1,0,0), \overrightarrow{v_{2}}=(0,-1,0)$ and $\overrightarrow{v_{3}}=$ $(0,0,0)$. These do not span $\mathbb{R}^{3}$ and since we have that $\overrightarrow{v_{3}}=\overrightarrow{0}$, these vectors are linearly dependent. The dimension of the column space and row space of $\lambda_{3}$ are both equal to the number of leading entries in the matrix which is 2 ; therefore the rank of $\lambda_{3}$ is also 2 .

To compute the eigenvalues we have the following:

$$
\operatorname{det}\left(\varphi I-\lambda_{3}\right)=(\varphi-1)(\varphi+1) \varphi=0
$$

Therefore, the roots or eigenvalues of this equation are $\varphi=-1,0,1$.

Finally, we say that $\vec{x} \in \mathbb{R}^{3}$, where $\vec{x} \neq \overrightarrow{0}$, is an eigenvector of $\lambda_{3}$ if it satisfies the equation $\lambda_{3} \vec{x}=\varphi \vec{x}$. We may set this equation up in the form of matrices as follows:

$$
\left[\begin{array}{ccc}
1 & 0 & 0 \\
0 & -1 & 0 \\
0 & 0 & 0
\end{array}\right]\left[\begin{array}{l}
x_{1} \\
x_{2} \\
x_{3}
\end{array}\right]=\varphi\left[\begin{array}{l}
x_{1} \\
x_{2} \\
x_{3}
\end{array}\right]
$$

Therefore, the solutions or eigenvectors satisfying this relation for $\varphi=-1,0,1$, respectively, are as follows:

$$
\overrightarrow{x_{1}}=\left[\begin{array}{l}
0 \\
1 \\
0
\end{array}\right], \overrightarrow{x_{2}}=\left[\begin{array}{l}
0 \\
0 \\
1
\end{array}\right], \overrightarrow{x_{3}}=\left[\begin{array}{l}
1 \\
0 \\
0
\end{array}\right]
$$




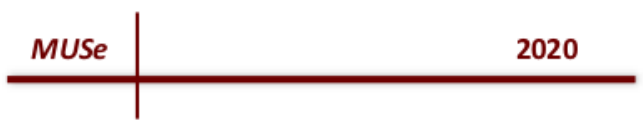

\section{Gell-Mann Matrix $\lambda_{4}$}

The fourth Gell-Mann matrix is a $3 \times 3$ square matrix that is symmetric due to $\lambda_{4}=\left(\lambda_{4}\right)^{T}$ and it is Hermitian due to $\lambda_{4}=\left(\lambda_{4}\right)^{*}$.

$$
\lambda_{4}=\left[\begin{array}{lll}
0 & 0 & 1 \\
0 & 0 & 0 \\
1 & 0 & 0
\end{array}\right]
$$

The determinant of $\lambda_{4}$ is equal to zero since it contains a row comprised of all zeros and the trace of $\lambda_{4}$ is also equal to zero.

Each of the three column vectors comprising $\lambda_{4}$ are $\overrightarrow{v_{1}}=(0,0,1), \overrightarrow{v_{2}}=(0,0,0)$ and $\overrightarrow{v_{3}}=$ $(1,0,0)$. These do not span $\mathbb{R}^{3}$ and since we have that $\overrightarrow{v_{2}}=\overrightarrow{0}$, these vectors are linearly dependent. The dimension of the column space and row space of $\lambda_{4}$ are both equal to the number of leading entries in the matrix which is 2 ; therefore the rank of $\lambda_{4}$ is also 2 .

To compute the eigenvalues we have the following:

$$
\begin{aligned}
\operatorname{det}\left(\varphi I-\lambda_{4}\right) & =\varphi\left|\begin{array}{ll}
\varphi & 0 \\
0 & \varphi
\end{array}\right|-0\left|\begin{array}{cc}
0 & 0 \\
-1 & \varphi
\end{array}\right|+(-1)\left|\begin{array}{cc}
0 & \varphi \\
-1 & 0
\end{array}\right| \\
& =\varphi\left(\varphi^{2}\right)-0-1(0-(-\varphi)) \\
& =\varphi^{3}-\varphi \\
& =\varphi\left(\varphi^{2}-1\right) \\
& =0
\end{aligned}
$$

Therefore, the roots or eigenvalues of this equation are $\varphi=-1,0,1$.

Finally, we say that $\vec{x} \in \mathbb{R}^{3}$, where $\vec{x} \neq \overrightarrow{0}$, is an eigenvector of $\lambda_{4}$ if it satisfies the equation $\lambda_{4} \vec{x}=\varphi \vec{x}$. We may set this equation up in the form of matrices as follows:

$$
\left[\begin{array}{lll}
0 & 0 & 1 \\
0 & 0 & 0 \\
1 & 0 & 0
\end{array}\right]\left[\begin{array}{l}
x_{1} \\
x_{2} \\
x_{3}
\end{array}\right]=\varphi\left[\begin{array}{l}
x_{1} \\
x_{2} \\
x_{3}
\end{array}\right]
$$

Therefore, the solutions or eigenvectors satisfying this relation for $\varphi=-1,0,1$, respectively, are as follows:

$$
\overrightarrow{x_{1}}=\left[\begin{array}{c}
-1 \\
0 \\
1
\end{array}\right], \overrightarrow{x_{2}}=\left[\begin{array}{l}
0 \\
1 \\
0
\end{array}\right], \overrightarrow{x_{3}}=\left[\begin{array}{l}
1 \\
0 \\
1
\end{array}\right]
$$

\section{Gell-Mann Matrix $\lambda_{5}$}

The fifth Gell-Mann matrix is a $3 \times 3$ square matrix that is antisymmetric due to $\lambda_{5}=-\left(\lambda_{5}\right)^{T}$ and it is Hermitian due to $\lambda_{5}=\left(\lambda_{5}\right)^{*}$. 


$$
\lambda_{5}=\left[\begin{array}{ccc}
0 & 0 & -i \\
0 & 0 & 0 \\
i & 0 & 0
\end{array}\right]
$$

The determinant of $\lambda_{5}$ is equal to zero since it contains a row comprised of all zeros and the trace of $\lambda_{5}$ is also equal to zero.

Each of the three column vectors comprising $\lambda_{5}$ are $\overrightarrow{v_{1}}=(0,0, i), \overrightarrow{v_{2}}=(0,0,0)$ and $\overrightarrow{v_{3}}=$ $(-i, 0,0)$. These do not span $\mathbb{R}^{3}$ and since we have that $\overrightarrow{v_{2}}=\overrightarrow{0}$, these vectors are linearly dependent. The dimension of the column space and row space of $\lambda_{5}$ are both equal to the number of leading entries in the matrix which is 2 ; therefore the rank of $\lambda_{5}$ is also 2 .

To compute the eigenvalues we have the following:

$$
\begin{aligned}
\operatorname{det}\left(\varphi I-\lambda_{5}\right) & =\varphi\left|\begin{array}{ll}
\varphi & 0 \\
0 & \varphi
\end{array}\right|-0\left|\begin{array}{cc}
0 & 0 \\
-i & \varphi
\end{array}\right|+i\left|\begin{array}{cc}
0 & \varphi \\
-i & 0
\end{array}\right| \\
& =\varphi\left(\varphi^{2}\right)-0+i(0-(-i \varphi)) \\
& =\varphi^{3}+i^{2} \varphi \\
& =\varphi^{3}-\varphi \\
& =\varphi\left(\varphi^{2}-1\right) \\
& =0
\end{aligned}
$$

Therefore, the roots or eigenvalues of this equation are $\varphi=-1,0,1$.

Finally, we say that $\vec{x} \in \mathbb{R}^{3}$, where $\vec{x} \neq \overrightarrow{0}$, is an eigenvector of $\lambda_{5}$ if it satisfies the equation $\lambda_{5} \vec{x}=\varphi \vec{x}$. We may set this equation up in the form of matrices as follows:

$$
\left[\begin{array}{ccc}
0 & 0 & -i \\
0 & 0 & 0 \\
i & 0 & 0
\end{array}\right]\left[\begin{array}{l}
x_{1} \\
x_{2} \\
x_{3}
\end{array}\right]=\varphi\left[\begin{array}{l}
x_{1} \\
x_{2} \\
x_{3}
\end{array}\right]
$$

Therefore, the solutions or eigenvectors satisfying this relation for $\varphi=-1,0,1$, respectively, are as follows:

$$
\overrightarrow{x_{1}}=\left[\begin{array}{l}
i \\
0 \\
1
\end{array}\right], \overrightarrow{x_{2}}=\left[\begin{array}{l}
0 \\
1 \\
0
\end{array}\right], \overrightarrow{x_{3}}=\left[\begin{array}{c}
-i \\
0 \\
1
\end{array}\right]
$$

\section{Gell-Mann Matrix $\lambda_{6}$}

The sixth Gell-Mann matrix is a $3 \times 3$ square matrix that is symmetric due to $\lambda_{6}=\left(\lambda_{6}\right)^{T}$ and it is Hermitian due to $\lambda_{6}=\left(\lambda_{6}\right)^{*}$.

$$
\lambda_{6}=\left[\begin{array}{lll}
0 & 0 & 0 \\
0 & 0 & 1 \\
0 & 1 & 0
\end{array}\right]
$$


The determinant of $\lambda_{6}$ is equal to zero since it contains a row comprised of all zeros and the trace of $\lambda_{6}$ is also equal to zero.

Each of the three column vectors comprising $\lambda_{6}$ are $\overrightarrow{v_{1}}=(0,0,0), \overrightarrow{v_{2}}=(0,0,1)$ and $\overrightarrow{v_{3}}=$ $(0,1,0)$. These do not span $\mathbb{R}^{3}$ and since we have that $\overrightarrow{v_{1}}=\overrightarrow{0}$, these vectors are linearly dependent. The dimension of the column space and row space of $\lambda_{6}$ are both equal to the number of leading entries in the matrix which is 2 ; therefore the rank of $\lambda_{6}$ is also 2 .

To compute the eigenvalues we have the following:

$$
\begin{aligned}
\operatorname{det}\left(\varphi I-\lambda_{6}\right) & =\varphi\left|\begin{array}{cc}
\varphi & -1 \\
-1 & \varphi
\end{array}\right|-0\left|\begin{array}{cc}
0 & -1 \\
0 & \varphi
\end{array}\right|+0\left|\begin{array}{cc}
0 & \varphi \\
0 & -1
\end{array}\right| \\
& =\varphi\left(\varphi^{2}-(1)\right)-0+0 \\
& =\varphi\left(\varphi^{2}-1\right) \\
& =0
\end{aligned}
$$

Therefore, the roots or eigenvalues of this equation are $\varphi=-1,0,1$.

Finally, we say that $\vec{x} \in \mathbb{R}^{3}$, where $\vec{x} \neq \overrightarrow{0}$, is an eigenvector of $\lambda_{6}$ if it satisfies the equation $\lambda_{6} \vec{x}=\varphi \vec{x}$. We may set this equation up in the form of matrices as follows:

$$
\left[\begin{array}{lll}
0 & 0 & 0 \\
0 & 0 & 1 \\
0 & 1 & 0
\end{array}\right]\left[\begin{array}{l}
x_{1} \\
x_{2} \\
x_{3}
\end{array}\right]=\varphi\left[\begin{array}{l}
x_{1} \\
x_{2} \\
x_{3}
\end{array}\right]
$$

Therefore, the solutions or eigenvectors satisfying this relation for $\varphi=-1,0,1$, respectively, are as follows:

$$
\overrightarrow{x_{1}}=\left[\begin{array}{c}
0 \\
-1 \\
1
\end{array}\right], \overrightarrow{x_{2}}=\left[\begin{array}{l}
1 \\
0 \\
0
\end{array}\right], \overrightarrow{x_{3}}=\left[\begin{array}{l}
0 \\
1 \\
1
\end{array}\right]
$$

\section{Gell-Mann Matrix $\lambda_{7}$}

The seventh Gell-Mann matrix is a $3 \times 3$ square matrix that is antisymmetric due to $\lambda_{7}=-\left(\lambda_{7}\right)^{T}$ and it is Hermitian due to $\lambda_{7}=\left(\lambda_{7}\right)^{*}$.

$$
\lambda_{7}=\left[\begin{array}{ccc}
0 & 0 & 0 \\
0 & 0 & -i \\
0 & i & 0
\end{array}\right]
$$

The determinant of $\lambda_{7}$ is equal to zero since it contains a row comprised of all zeros and the trace of $\lambda_{7}$ is also equal to zero.

Each of the three column vectors comprising $\lambda_{7}$ are $\overrightarrow{v_{1}}=(0,0,0), \overrightarrow{v_{2}}=(0,0, i)$ and $\overrightarrow{v_{3}}=$ $(0,-i, 0)$. These do not span $\mathbb{R}^{3}$ and since we have that $\overrightarrow{v_{1}}=\overrightarrow{0}$, these vectors are linearly dependent. The dimension of the column space and row space of $\lambda_{7}$ are both equal to the number of leading entries in the matrix which is 2 ; therefore the rank of $\lambda_{7}$ is also 2 . 


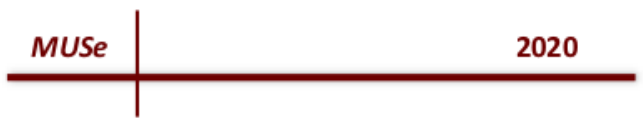

To compute the eigenvalues we have the following:

$$
\begin{aligned}
\operatorname{det}\left(\varphi I-\lambda_{7}\right) & =\varphi\left|\begin{array}{cc}
\varphi & i \\
-i & \varphi
\end{array}\right|-0\left|\begin{array}{cc}
0 & i \\
0 & \varphi
\end{array}\right|+0\left|\begin{array}{cc}
0 & \varphi \\
0 & -i
\end{array}\right| \\
& =\varphi\left(\varphi^{2}-\left(-i^{2}\right)\right)-0+0 \\
& =\varphi\left(\varphi^{2}-(1)\right) \\
& =\varphi\left(\varphi^{2}-1\right) \\
& =0
\end{aligned}
$$

Therefore, the roots or eigenvalues of this equation are $\varphi=-1,0,1$.

Finally, we say that $\vec{x} \in \mathbb{R}^{3}$, where $\vec{x} \neq \overrightarrow{0}$, is an eigenvector of $\lambda_{7}$ if it satisfies the equation $\lambda_{7} \vec{x}=\varphi \vec{x}$. We may set this equation up in the form of matrices as follows:

$$
\left[\begin{array}{ccc}
0 & 0 & 0 \\
0 & 0 & -i \\
0 & i & 0
\end{array}\right]\left[\begin{array}{l}
x_{1} \\
x_{2} \\
x_{3}
\end{array}\right]=\varphi\left[\begin{array}{l}
x_{1} \\
x_{2} \\
x_{3}
\end{array}\right]
$$

Therefore, the solutions or eigenvectors satisfying this relation for $\varphi=-1,0,1$, respectively, are as follows:

$$
\overrightarrow{x_{1}}=\left[\begin{array}{l}
0 \\
i \\
1
\end{array}\right], \overrightarrow{x_{2}}=\left[\begin{array}{l}
1 \\
0 \\
0
\end{array}\right], \overrightarrow{x_{3}}=\left[\begin{array}{c}
0 \\
-i \\
1
\end{array}\right]
$$

\section{Gell-Mann Matrix $\lambda_{8}$}

The eighth and final Gell-Mann matrix is a $3 \times 3$ square matrix that is diagonal due to every entry being zero other than on the main diagonal and it is Hermitian due to $\lambda_{8}=\left(\lambda_{8}\right)^{*}$.

$$
\lambda_{8}=\left[\begin{array}{ccc}
\frac{1}{\sqrt{3}} & 0 & 0 \\
0 & \frac{1}{\sqrt{3}} & 0 \\
0 & 0 & \frac{-2}{\sqrt{3}}
\end{array}\right]
$$

The determinant of $\lambda_{8}$ is equal to the product of all entries on the main diagonal which is $\frac{1}{\sqrt{3}} \cdot \frac{1}{\sqrt{3}} \cdot \frac{-2}{\sqrt{3}}=\frac{-2}{3 \sqrt{3}}$ and the trace of $\lambda_{8}$ is equal to zero.

Each of the three column vectors comprising $\lambda_{8}$ are $\overrightarrow{v_{1}}=\left(\frac{1}{\sqrt{3}}, 0,0\right), \overrightarrow{v_{2}}=\left(0, \frac{1}{\sqrt{3}}, 0\right)$ and $\overrightarrow{v_{3}}=$ $\left(0,0, \frac{-2}{\sqrt{3}}\right)$. These do span $\mathbb{R}^{3}$ and are linearly independent due to the fact that there do not exist scalars $c_{1}, c_{2}, c_{3}$ not all equal zero such that:

$$
\sum_{i=1}^{3} c_{i} \overrightarrow{v_{i}}=c_{1} \overrightarrow{v_{1}}+c_{2} \overrightarrow{v_{2}}+c_{3} \overrightarrow{v_{3}}=0
$$

The dimension of the column space and row space of $\lambda_{8}$ are both equal to the number of leading entries in the matrix which is 3 ; therefore the rank of $\lambda_{8}$ is also 3 . 


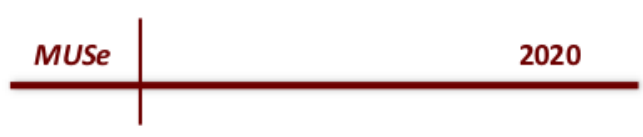

To compute the eigenvalues we have the following:

$$
\operatorname{det}\left(\varphi I-\lambda_{8}\right)=\left(\varphi-\frac{1}{\sqrt{3}}\right)\left(\varphi-\frac{1}{\sqrt{3}}\right)\left(\varphi+\frac{2}{\sqrt{3}}\right)=0
$$

Therefore, the roots or eigenvalues of this equation are $\varphi=\frac{1}{\sqrt{3}}, \frac{1}{\sqrt{3}}, \frac{-2}{\sqrt{3}}$.

Finally, we say that $\vec{x} \in \mathbb{R}^{3}$, where $\vec{x} \neq \overrightarrow{0}$, is an eigenvector of $\lambda_{8}$ if it satisfies the equation $\lambda_{8} \vec{x}=\varphi \vec{x}$. We may set this equation up in the form of matrices as follows:

$$
\left[\begin{array}{ccc}
\frac{1}{\sqrt{3}} & 0 & 0 \\
0 & \frac{1}{\sqrt{3}} & 0 \\
0 & 0 & \frac{-2}{\sqrt{3}}
\end{array}\right]\left[\begin{array}{l}
x_{1} \\
x_{2} \\
x_{3}
\end{array}\right]=\varphi\left[\begin{array}{l}
x_{1} \\
x_{2} \\
x_{3}
\end{array}\right]
$$

Therefore, the solutions or eigenvectors satisfying this relation for $\varphi=\frac{1}{\sqrt{3}}, \frac{1}{\sqrt{3}}, \frac{-2}{\sqrt{3}}$, respectively, are as follows:

$$
\overrightarrow{x_{1}}=\left[\begin{array}{l}
1 \\
0 \\
0
\end{array}\right], \overrightarrow{x_{2}}=\left[\begin{array}{l}
0 \\
1 \\
0
\end{array}\right], \overrightarrow{x_{3}}=\left[\begin{array}{l}
0 \\
0 \\
1
\end{array}\right]
$$

\section{Quantum Chromodynamics}

Quantum chromodynamics (QCD) is the theory behind one of the four forces of nature: the strong nuclear force. The main particle constituents of this theory involve quarks, which come in six different 'flavours', and gluons. Quarks are the elementary particles making up protons and neutrons in the atomic nucleus and the six flavours are up $(u)$, down $(d)$, charm $(c)$, strange $(s)$, top $(t)$ and bottom $(b)$ [6]. Gluons are a specific type of particle called a boson, which are known as "force carriers" [5].

Protons and neutrons in the atomic nucleus are known as strongly interacting particles and gluons are responsible for "gluing" the nucleons to each other and keep the atomic nucleus from flying apart. Just as important, gluons are also responsible for keeping the quarks constituting a nucleon together.

During the $20^{\text {th }}$ century, many strongly interacting particles were discovered by various methods and it seemed apparent to physicists that they were not all fundamental particles. Murray Gell-Mann was one of the physicists who proposed a way of bringing all these particles together under one umbrella and further dividing them up based on specific properties such as spin, mass and charge [6]. The model Gell-Mann came up with was called the quark model and group theory was very useful when applied to it; specifically, the Lie group $S U_{3}(\mathbb{C})$ became the symmetry group of quantum chromodynamics [6].

The quarks were organized into three families consisting of the up and down quarks, the top and bottom quarks, and the charm and strange quarks [5]. This quark model was able to simply classify the amazing variety of strongly interacting particles and showed that all these particles 
came in only a few specific combinations. A baryon is a particle composed of three quarks and a meson is a particle composed of a quark and an anti-quark [6].

To satisfy certain aspects of the theory of QCD, quarks needed a new label called a quantum number to accurately describe them; this quantum number has became known as colour. The colour charge associated to a quark is either red, blue or green and this group of colours can also be described in terms of $S_{3}$. It is also the same story for anti-quarks, which have either anti-red, anti-blue or anti-green as a colour charge; in this case, of course, we have anti-colour. It is noted in the reference [6] by Kim Milton that this $S U_{3}$ group of colour has no relation to the flavour group $S_{3}$; however, one can explore the flavour aspect in many particle physics books. It should also be noted that when speaking of colour it is not a reference to what we see around us in our everyday lives.

\subsection{Quarks and Colour Charge}

Now, let us look a little deeper into the mathematics of quark and gluon colour. Quarks do not interact with each other directly, but do so via gluons, and each flavour of quark also has an associated colour as previously discussed [6]. Since we want $S U_{3}(\mathbb{C})$ to play a role in the theory of this particle interaction, let us assume we can assign the colour of each quark to a $3 \times 1$ column vector, since $S U_{3}$ can act on elements of a three dimensional complex vector space by matrix multiplication. These three colour column vectors act as a basis for the three dimensional vector space.

$$
\text { red }(r)=\left[\begin{array}{l}
1 \\
0 \\
0
\end{array}\right] \text {, blue }(b)=\left[\begin{array}{l}
0 \\
1 \\
0
\end{array}\right] \text {, green }(g)=\left[\begin{array}{l}
0 \\
0 \\
1
\end{array}\right]
$$

By letting an element $M \in S U_{3}$ act on one of the colours $c=r, b$ or $g$, we obtain another $3 \times 1$ matrix corresponding to the new quark colour $c^{\prime}=r^{\prime}, b^{\prime}, g^{\prime}$ under the transformation.

$$
\begin{aligned}
& M \cdot r=M r=r^{\prime} \\
& M \cdot b=M b=b^{\prime} \\
& M \cdot g=M g=g^{\prime}
\end{aligned}
$$

We are able to put all colours together into one column vector and have an element $M$ act on it by left multiplication as shown below:

$$
c \rightarrow M c=\left[\begin{array}{lll}
m_{11} & m_{12} & m_{13} \\
m_{21} & m_{22} & m_{23} \\
m_{31} & m_{32} & m_{33}
\end{array}\right]\left[\begin{array}{l}
r \\
b \\
g
\end{array}\right]=c^{\prime}
$$

Note that a colour transformation via the identity element of $\mathrm{SU}_{3}$ will leave the original colour unchanged; this is the trivial colour transformation. Also, since there are 6 flavours of quarks and 3 colour charge possibilities, we have a total of 18 different types of quarks. 


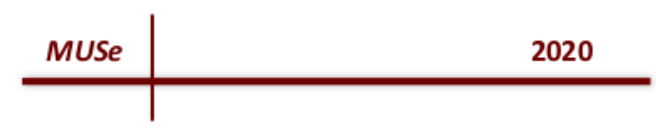

It is also possible that the elements of the three dimensional complex vector space are $1 \times 3$ row vectors with complex entries. We can assign an anti-colour to each of these row vectors in the same way as we assigned a colour to each column vector; therefore, we have the following:

$$
\operatorname{anti-red}(\bar{r})=\left[\begin{array}{lll}
1 & 0 & 0
\end{array}\right], \text { anti-blue }(\bar{b})=\left[\begin{array}{lll}
0 & 1 & 0
\end{array}\right] \text {, anti-green }(\bar{g})=\left[\begin{array}{lll}
0 & 0 & 1
\end{array}\right]
$$

Accelerator experiments and cosmic ray observations in the middle of the $20^{\text {th }}$ century have confirmed the existence of anti-particles for most types, including quarks [6]. In our case each anticolour can correspond to the property carried by various flavours of anti-quarks. An element $M \in$ $S_{3}$ can act on each row vector and we would then obtain another $1 \times 3$ row vector corresponding to a new anti-colour.

$$
\begin{aligned}
& \bar{r} \cdot M=\bar{r} M=\bar{r}^{\prime} \\
& \bar{b} \cdot M=\bar{b} M=\bar{b}^{\prime} \\
& \bar{g} \cdot M=\bar{g} M=\bar{g}^{\prime}
\end{aligned}
$$

We can combine all the anti-colours together into one colour vector and have an element $M$ act on it by right matrix multiplication as shown below:

$$
\bar{c} \rightarrow \bar{c} M=\left[\begin{array}{lll}
\bar{r} & \bar{b} & \bar{g}
\end{array}\right]\left[\begin{array}{lll}
m_{11} & m_{12} & m_{13} \\
m_{21} & m_{22} & m_{23} \\
m_{31} & m_{32} & m_{33}
\end{array}\right]=\bar{c}^{\prime}
$$

Once again, a transformation to a new anti-colour via the identity element would leave the original anti-colour row vector unchanged.

As stated before, any two quarks need gluons to be the intermediary between them to process an interaction. Since protons have been observed to have no colour charge, the net colour charge must be equal to zero. Just as two electric charges, such as $\frac{1}{2}$ and $\frac{-1}{2}$, are zero when added together, we can add red, blue and green quarks together in baryons to make a net zero colour charge; in other words, the nucleon is "colourless" [3].

The other quark grouping possibility is called a meson. These particles are constituted of a quark and an anti-quark and they also have been shown to have no colour charge. As discussed before, a quark has a colour property and likewise an anti-quark has an anti-colour property. The same analogy to electric charge also can explain why a green quark and an anti-green anti-quark become a "colourless" meson. A colour and its anti-colour are able to cancel each other out and following this same line of thinking, a quark and anti-quark would also cancel each other out. This close proximity of antagonistic particles is one property that leads to the meson having a very short life span.

\subsection{Quantum Superposition and Colourless Particles}

There is a principle of quantum mechanics called superposition which says that any number of quantum states may be added together or "superposed" to become an equally valid quantum state [1]. In our case of colour, and in particular we can take the meson as an example, we can 
say that each possible colour combination a meson can achieve, such as blue and anti-blue quark combination, is a quantum state. We know that a meson has two other possible quantum states and therefore we may add all these together to obtain an equally valid quantum state [1]. We can see this using matrices as outlined below:

$$
\begin{gathered}
\text { Meson }=r \bar{r}+b \bar{b}+g \bar{g}=\left[\begin{array}{l}
1 \\
0 \\
0
\end{array}\right]\left[\begin{array}{lll}
1 & 0 & 0
\end{array}\right]+\left[\begin{array}{l}
0 \\
1 \\
0
\end{array}\right]\left[\begin{array}{lll}
0 & 1 & 0
\end{array}\right]+\left[\begin{array}{l}
0 \\
0 \\
1
\end{array}\right]\left[\begin{array}{lll}
0 & 0 & 1
\end{array}\right] \\
\text { Meson }=\left[\begin{array}{lll}
1 & 0 & 0 \\
0 & 0 & 0 \\
0 & 0 & 0
\end{array}\right]+\left[\begin{array}{lll}
0 & 0 & 0 \\
0 & 1 & 0 \\
0 & 0 & 0
\end{array}\right]+\left[\begin{array}{lll}
0 & 0 & 0 \\
0 & 0 & 0 \\
0 & 0 & 1
\end{array}\right]=\left[\begin{array}{lll}
1 & 0 & 0 \\
0 & 1 & 0 \\
0 & 0 & 1
\end{array}\right]
\end{gathered}
$$

Therefore, we can see that the identity matrix of $S U_{3}(\mathbb{C})$ corresponds to the colourless meson with the three superposed colours. We may note that since each quantum state has an equal probability of showing up we could add a factor of $\frac{1}{3}$ to this and we would get $(r \bar{r}+b \bar{b}+g \bar{g}) / 3$.

We can now assume that the same approach will also work for a baryon, since they are also composed of quarks with colour charge. As mentioned in the last section, baryons must also exhibit a colourless property like the meson, and therefore each of its three quarks must be a different colour, choosing from red, blue and green.

In our example concerning mesons, each colour anti-colour pair could be expressed as a $3 \times 3$ matrix which were added together to create the meson with the superposed colour combination. Therefore, let us assume we can do something similar for a baryon by letting each quantum state correspond to a $3 \times 3$ matrix in our three dimensional complex vector space.

First, we must establish every combination of colours we can have, assuming that we can distinguish between individual quarks. We can label the quarks by $q_{j} ; j \in\{1,2,3\}$, and then choose a colour for $q_{1}$, say red; so $q_{1}=r$. Next, we go onto $q_{2}$ and choose a colour that has not been chosen yet; in this case we make $q_{2}=b$. Finally, by process of elimination, we choose $q_{3}=g$; this makes each quark a different colour, i.e. assigns a different quantum state to each quark, giving a specific quantum state for the baryon.

We can continue this process to obtain all possible colour combinations using the three colours for the three quarks to obtain every quantum state possible for a baryon. We can see that it is simply $3 !=6$ possible combinations or quantum states, as outlined in matrix form below:

$$
\begin{gathered}
\text { Baryon }=r b g+r g b+b r g+b g r+g r b+g b r \\
\text { Baryon }=\left[\begin{array}{lll}
1 & 0 & 0 \\
0 & 1 & 0 \\
0 & 0 & 1
\end{array}\right]+\left[\begin{array}{lll}
1 & 0 & 0 \\
0 & 0 & 1 \\
0 & 1 & 0
\end{array}\right]+\left[\begin{array}{lll}
0 & 1 & 0 \\
1 & 0 & 0 \\
0 & 0 & 1
\end{array}\right]+\left[\begin{array}{lll}
0 & 0 & 1 \\
1 & 0 & 0 \\
0 & 1 & 0
\end{array}\right]+\left[\begin{array}{lll}
0 & 1 & 0 \\
0 & 0 & 1 \\
1 & 0 & 0
\end{array}\right]+\left[\begin{array}{lll}
0 & 0 & 1 \\
0 & 1 & 0 \\
1 & 0 & 0
\end{array}\right]
\end{gathered}
$$




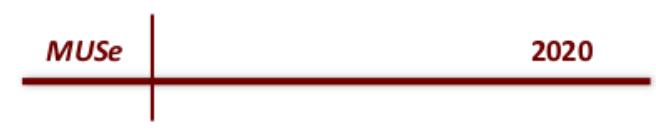

Also note we can add a factor of $\frac{1}{6}$ to this equation since each of the six quantum states have an equal probability of being achieved. Therefore, our equation above should be $(r b g+r g b+b r g+$ $b g r+g r b+g b r) / 6$.

Notice that in equation 5.11 we can rewrite this formula to have a basis composed of the three colour vectors. We can do this since we are in a three dimensional complex vector space; or in our case a three dimensional colour space.

$$
\begin{aligned}
\text { Baryon } & =\frac{r b g+r g b+b r g+b g r+g r b+g b r}{6} \\
& =\frac{(b g+g b) r}{6}+\frac{(r g+g r) b}{6}+\frac{(r b+b r) g}{6} \\
& =\frac{(b g+g b)}{2}\left[\begin{array}{l}
1 \\
0 \\
0
\end{array}\right] \frac{1}{3}+\frac{(r g+g r)}{2}\left[\begin{array}{l}
0 \\
1 \\
0
\end{array}\right] \frac{1}{3}+\frac{(r b+b r)}{2}\left[\begin{array}{l}
0 \\
0 \\
1
\end{array}\right] \frac{1}{3} \\
& =\frac{(b g+g b)}{2}\left[\begin{array}{c}
1 / 3 \\
0 \\
0
\end{array}\right]+\frac{(r g+g r)}{2}\left[\begin{array}{c}
0 \\
1 / 3 \\
0
\end{array}\right]+\frac{(r b+b r)}{2}\left[\begin{array}{c}
0 \\
0 \\
1 / 3
\end{array}\right]
\end{aligned}
$$

Therefore, the above equation still corresponds to a colourless baryon, even if it looks much different than our first straightforward equation.

The last line of Equation 5.9 above is a quantum state and also shows that the baryon is composed of up to three separate quantum states based upon each vector in the colour basis. When these are added together we get an equally valid quantum state and this includes all possible combinations of individual quarks and their colours composing a suitable baryon.

\subsection{Gluons and Colour Charge}

The interior of a nucleon not only contains quarks but holding them together are particles called gluons. It would be reasonable to make a conjecture that since gluons are responsible for the strong interaction between quarks, that they are also responsible for sending information about a quark's colour to the other quarks. This would make gluons a part of the representation of $S U_{3}(\mathbb{C})$ since the group acts on colour vectors of quarks and produces a new colour charge. Since a necessary property is that all quarks have to be different colours in a baryon, the gluons would then have to send the information of a change in the colour of one quark to the others; this in turn would change the colour of the quark which is equal to the new colour of the first. This process would then repeat until all quarks were different colours again; however, there could be external interactions causing colour change that would warrant this process to have no foreseeable stopping point.

A point must be explored from our previous discussion on quark colour. The identity of $S_{3}$ is trivial and causes no change to the colour of the quark in question. Therefore, if we assume that there is a gluon corresponding to the identity matrix, this gluon would have no real effect and cause no transformation. Therefore, we could assume that there is no "identity gluon"; in fact 


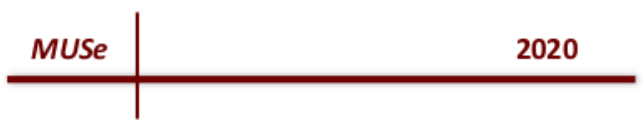

experiments have found that there are only 8 gluons, and there is no reason to believe that a gluon exists for which there is no purpose [11].

From the fact that the identity matrix is not used, we can assume that gluons are still a part of the representation of $S U_{3}$ but more explicitly contained in the Lie algebra $\mathfrak{s u}_{3}$. This is due to the fact that all matrices in the Lie algebra of $S U_{3}$ have trace equal to zero.

A simple way to think about the gluons is to assign each type of gluon to a basis element of $\mathfrak{s u}_{3}$, which we earlier found to be exactly the Gell-Mann matrices, $\lambda_{i} ; i \in\{1,2, \ldots, 8\}$. Assigning them in this manner allows us to describe all the types of gluons since each Gell-Mann matrix is linearly independent from the next.

Since gluons are responsible for colour changes in quarks, perhaps gluons also have colour in order to have a physical interaction in the real world. From multiple references it has been said that the gluons each carry a colour and an anti-colour, however they do so with a certain level of probability. Since nothing can be quite certain in the quantum realm, the gluon assigned to a Gell-Mann matrix has an equal probability of carrying a specific colour and anti-colour or another.

We can see this in an example by taking a Gell-Mann matrix, say $\lambda_{6}$ :

$$
\lambda_{6}=\left[\begin{array}{lll}
0 & 0 & 0 \\
0 & 0 & 1 \\
0 & 1 & 0
\end{array}\right]
$$

By looking at each nonzero entry in the manner we did before we can see that each one corresponds to both a colour and anti-colour. Entry $a_{i j}=a_{32}=1$ corresponds to green, anti-blue and entry $a_{i j}=a_{23}=1$ corresponds to blue, anti-green. Interestingly, these entries, when taken in terms of colour, are opposites of each other.

We can also use our colour column and row vectors to act on each gluon by conjugation, $\lambda_{i} \mapsto \bar{c} \cdot \lambda_{i} \cdot c ; \forall i \in\{1,2, \ldots, 8\}$. We can use $\lambda_{6}$ as our example gluon again, shown below:

$$
\left[\begin{array}{lll}
0 & 0 & 0 \\
0 & 0 & 1 \\
0 & 1 & 0
\end{array}\right] \mapsto\left[\begin{array}{lll}
\bar{r} & \bar{b} & \bar{g}
\end{array}\right]\left[\begin{array}{lll}
0 & 0 & 0 \\
0 & 0 & 1 \\
0 & 1 & 0
\end{array}\right]\left[\begin{array}{l}
r \\
b \\
g
\end{array}\right]=\left[\begin{array}{lll}
0 & \bar{g} & \bar{b}
\end{array}\right]\left[\begin{array}{l}
r \\
b \\
g
\end{array}\right]=[\bar{g} b+\bar{b} g]
$$

The final result is a $1 \times 1$ matrix with an entry that directly agrees with our first interpretation of the $\lambda_{6}$ gluon and the possible colour and anti-colour that it carries. Therefore, we can use either method to acquire the information of a gluons colour property and apply them to the rest of the Gell-Mann matrices, which we can see on the following page.

$$
\lambda_{1}=\left[\begin{array}{lll}
0 & 1 & 0 \\
1 & 0 & 0 \\
0 & 0 & 0
\end{array}\right]=[\text { blue anti-red, red anti-blue }]
$$




$$
\begin{aligned}
& \left.\lambda_{2}=\left[\begin{array}{ccc}
0 & -i & 0 \\
i & 0 & 0 \\
0 & 0 & 0
\end{array}\right]=-i\left[\begin{array}{ccc}
0 & 1 & 0 \\
-1 & 0 & 0 \\
0 & 0 & 0
\end{array}\right]=-i[- \text { (blue anti-red }), \text { red anti-blue }\right] \\
& \lambda_{3}=\left[\begin{array}{ccc}
1 & 0 & 0 \\
0 & -1 & 0 \\
0 & 0 & 0
\end{array}\right]=[\text { red anti-red, }-(\text { blue anti-blue })] \\
& \lambda_{4}=\left[\begin{array}{lll}
0 & 0 & 1 \\
0 & 0 & 0 \\
1 & 0 & 0
\end{array}\right]=[\text { red anti-green, green anti-red }] \\
& \lambda_{5}=\left[\begin{array}{ccc}
0 & 0 & -i \\
0 & 0 & 0 \\
i & 0 & 0
\end{array}\right]=-i\left[\begin{array}{ccc}
0 & 0 & 1 \\
0 & 0 & 0 \\
-1 & 0 & 0
\end{array}\right]=-i[\text { red anti-green, }-(\text { green anti-red })] \\
& \left.\lambda_{6}=\left[\begin{array}{lll}
0 & 0 & 0 \\
0 & 0 & 1 \\
0 & 1 & 0
\end{array}\right]=\text { [blue anti-green, green anti-blue }\right] \\
& \lambda_{7}=\left[\begin{array}{ccc}
0 & 0 & 0 \\
0 & 0 & -i \\
0 & i & 0
\end{array}\right]=-i\left[\begin{array}{ccc}
0 & 0 & 0 \\
0 & 0 & 1 \\
0 & -1 & 0
\end{array}\right]=-i[\text { blue anti-green, }-(\text { green anti-blue })]
\end{aligned}
$$

Since each colour anti-colour pair has an equal probability of being the "true" property of the specific gluon in question, we can simply state them in a list of equations, shown below in the same order as above:

$$
\begin{gathered}
\lambda_{1}=\frac{(r \bar{b}+b \bar{r})}{2}, \lambda_{2}=-i\left(\frac{r \bar{b}-b \bar{r}}{2}\right), \lambda_{3}=\frac{(r \bar{r}-b \bar{b})}{2}, \lambda_{4}=\frac{(r \bar{g}+g \bar{r})}{2} \\
\lambda_{5}=-i\left(\frac{r \bar{g}-g \bar{r}}{2}\right), \lambda_{6}=\frac{(b \bar{g}+g \bar{b})}{2}, \lambda_{7}=-i\left(\frac{b \bar{g}-g \bar{b}}{2}\right), \lambda_{8}=\frac{1}{\sqrt{3}}\left(\frac{r \bar{r}+b \bar{b}-2 g \bar{g}}{3}\right)
\end{gathered}
$$

One can see above that these matrices do not take on a form that would suggest they would be colourless, such as in our examples previously on the meson and baryon. Therefore, we can say that there are no colourless gluons. 


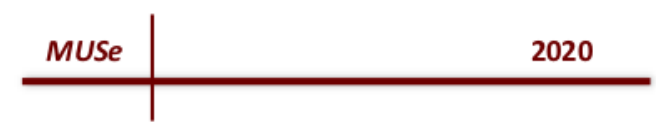

\section{Conclusion}

The main purpose of this report was to explore the Lie theory and group theory of the group $S U_{3}(\mathbb{C})$ and its Lie algebra $\mathfrak{s u}_{3}(\mathbb{C})$, and then to use that information in introducing and exploring a part of quantum field theory known as quantum chromodynamics or QCD.

First, we looked at the lower dimensional case of the Lie group $S U_{2}(\mathbb{C})$ and used the Pauli matrices as a set of generators for the group. This allowed the reader to gain some insight into the process we would be taking with the larger group $S_{3}$ and the Gell-Mann matrices later on. As stated in the introduction, if one wants to understand something complex, they should first look at an example of a similar problem in which things are a little simpler. In this simpler case, it was shown that using the elements of the Lie algebra with a chosen basis, we could obtain elements of the Lie group which subsequently also had the same basis as a set of generators for the group itself.

A number of pages were also dedicated to the properties specific to each Gell-Mann matrix. Sources explaining the physics of quantum field theory, which explicitly used the Gell-Mann matrices in calculations and discussions, never went into the specific mathematics of the matrices. The types of items that were covered in this report included basic matrix properties, finding the eigenvalues and eigenvectors, and as well looking at the dimension, rank and linear independence of each matrix's column vectors. These topics are outlined in such a way that it could be a useful reference for those studying related areas.

Finally, it was shown that we can actually use the Gell-Mann matrices to talk about gluons and quarks and their property of colour charge. The Lie group or symmetry group $S U_{3}(\mathbb{C})$ plays a major role in the certain type of quantum field theory known as quantum chromodynamics and is extremely useful in explaining many aspects of it. QCD includes knowledge of quantum mechanics, quantum field theory and grand unified theories. For the interested reader, one could look up such authors as Edward Witten, Steven Weinberg, Peter Woit, Paul Dirac or Richard Feynman and Murray Gell-Mann to name but a few.

There are still many problems with quantum chromodynamics such as asymptotic freedom, and why physicists have not discovered quarks or gluons that are separate or free particles [10]. Quarks and gluons that are all on their own in nature have not been found as of yet. This property has to do with the fact that the farther apart two quarks are, the strength of the strong interaction increases. This is the exact opposite of, for example, the gravitational force which diminishes in strength the farther apart two objects are from each other.

The Standard Model of particle physics has done a very good job of organizing and describing all particles and forces that physicists and scientists in general have come across in our natural world (other than gravity) by way of cosmic ray experiments and particle accelerators. The recent success of the Large Hadron Collider in Europe is very promising as pieces of the cosmic puzzle are slowly filled in, such as the discovery of the Higgs boson.

It is hopeful that the scientific community will continue to have success in the future and physicists as a whole will keep the field of mathematics very close. Keeping open communication 


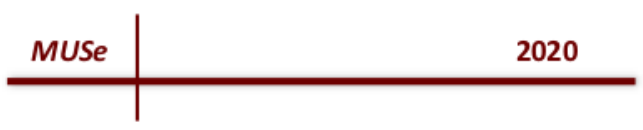

between these two entities seems to be inevitable while humans continue in their search of all the properties of the universe.

\section{References}

[1] Sheldon L. Glashow. From Alchemy to Quarks. Brooks/Cole Publishing Company, Wadsworth, Inc., 1994. Page 633. Print.

[2] Ian Grojnowski. Representation Theory. Timothy Gowers (Editor). The Princeton Companion to Mathematics. Princeton University Press, 2008. Pages 419 - 431. Print.

[3] Don Lincoln. Understanding the Universe: From Quarks to the Cosmos. World Scientific Publishing Co. Pte. Ltd., 2004. Pages 118 - 120. Print.

[4] Holger Lyre. Gauge Symmetry. Daniel Greenberger, Klaus Hentschel, Friedel Weinert (Editors). Compendium of Quantum Physics: Concepts, Experiments, History and Philosophy. Springer Dordrecht Heidelberg London New York. Springer- Verlag Berlin Heidelberg 2009. Pages 248 - 255. Print.

[5] Kim Milton. Particle Physics. Daniel Greenberger, Klaus Hentschel, Friedel Weinert (Editors). Compendium of Quantum Physics: Concepts, Experiments, History and Philosophy. Springer Dordrecht Heidelberg London New York. Springer- Verlag Berlin Heidelberg 2009. Pages 455 - 459. Print.

[6] Kim Milton. Quantum Chromodynamics (QCD). Daniel Greenberger, Klaus Hentschel, Friedel Weinert (Editors). Compendium of Quantum Physics: Concepts, Experiments, History and Philosophy. Springer- Verlag Berlin Heidelberg 2009. Pages 524 - 527. Print.

[7] Harriet Pollatsek. Lie Groups: A Problem-Oriented Introduction via Matrix Groups. The Mathematical Association of America Inc. United States of America, 2009. Print.

[8] Todd Rowland. Lie Group. From MathWorld-A Wolfram Web Resource, created by Eric W. Weisstein. http://mathworld.wolfram.com/LieGroup.html.

[9] Todd Rowland. Manifold. From MathWorld-A Wolfram Web Resource, created by Eric W. Weisstein. http://mathworld.wolfram.com/Manifold.html.

[10] Steven Weinberg. The Search for Unity: Notes for a History of Quantum Field Theory. Daedalus, Vol. 106, No. 4, Discoveries and Interpretations: Studies in Contemporary Scholarship, Volume II (Fall, 1977), pp. 17-35. The MIT Press, 1977. Print.

[11] Steven Weinberg. The Discovery of Subatomic Particles. Cambridge University Press, Cambridge, United Kingdom. Revised Edition Published 2003. Pages 155 - 157. Print.

[12] Eric W. Weisstein. Kronecker Delta. From MathWorld-A Wolfram Web Resource. http://mathworld.wolfram.com/KroneckerDelta.html.

[13] Eric W. Weisstein. Smooth Manifold. From MathWorld-A Wolfram Web Resource. http://mathworld.wolfram.com/SmoothManifold.html.

[14] Peter Woit. Quantum Theory, Groups and Representations: An Introduction (under construction). Department of Mathematics, Columbia University, 2016. 